\title{
Interpretation of meV Resolution Phonon EELS Data
}

\author{
P.E. Batson ${ }^{1}$, M.J. Lagos ${ }^{2}$ \\ ${ }^{1}$ Department of Physics and Astronomy, Rutgers University, NJ, USA \\ ${ }^{2}$ Department of Materials Science and Engineering, McMaster University, Ontario, Canada
}

With the introduction of the Nion Co. Hermes high resolution monochromator, sub-10 meV resolution EELS has become a reality for electron microscopy.[1] This has enabled an IRlike spectroscopy in aloof scattering,[2] and mapping of bulk and surface phonon modes in a single $\mathrm{MgO}$ nanocube.[3] Theoretical work has also begun.[4] However, below about $50 \mathrm{meV}$, where the phonon systems must be treated with a scattering theory at finite temperature our understanding is still developing. [5] There, we expect Boson occupation statistics to affect the observed EELS results, giving both energy gain, and modification of energy loss results. There turn out to be three kinds of scattering events: 1) spontaneous emission of a collective phonon by the probe, an energy loss process, 2) an induced emission by occupied phonon modes, also an energy loss process, and 3) an induced absorption, or electron energy gain. We begin by writing the scattering probability for EELS in the familiar dielectric form, or equivalently, using the Dynamical Form factor, $S(\mathbf{q}, \omega)[5]$

$$
P(\mathbf{q}, \omega)=\frac{8 \pi e^{2}}{q^{2}} \operatorname{Im}\left(-\frac{1}{\epsilon(\mathbf{q}, \omega)}\right)=2 \pi\left(\frac{4 \pi e^{2}}{q^{2}}\right)^{2} S(\mathbf{q}, \omega)
$$

with the form factor written to remind us of its relationship the Fermi's Golden Rule:

$$
S(\mathbf{q}, \omega)=Z^{-1} \sum_{m n} e^{-\beta E_{m}}\left|\left(\rho_{q}^{\dagger}\right)_{n m}\right|^{2} \delta\left(\omega-\omega_{m n}\right) \text { with } Z=\sum_{m} e^{-\beta E_{m}}
$$

where $\left|\left(\rho_{q}^{\dagger}\right)_{n m}\right|^{2}$ is a matrix element that defines overlapping specimen charge density fluctuations, and the product of this matrix element and the Density of States in energy is the quantity that is of interest for understanding the ground state of the material. At finite temperature, there are many occupied initial states $<m \mid$ weighted by the Boltzmann factor $e^{-\beta E_{m}}$ contributing to a distortion of the Phonon Density of States that one would like to measure. Finally, we find that the the statistical modification of the spectrum becomes

$$
(1+<n>) \delta\left(\omega-\omega_{p h}\right) / \omega_{p h}+<n>\delta\left(\omega+\omega_{p h}\right) / \omega_{p h} \text { with }<n>=\frac{1}{\left(e^{\beta \omega_{p h}}-1\right)} .
$$

The first term here accounts for the spontaneous and induced energy loss. The second term reflects induced energy gain. While this result seems minor, its influence on the measured spectra is profound. Below, we compare the ZLP and Bulk data in Fig. 1. In Fig 2. we subtract the ZLP from the bulk data, revealing two strong peaks on either side of zero. We find elsewhere in this proceedings, that these peaks obey the Principle of Detailed Balance, that defines the exchange of energy between two processes in thermal equilibrium. [5] In Fig. 3, we compare the residual peaks with expected statistical modification from by Eqn. (3) above. In Fig. 4. we correct the raw subtracted data by removal of the predicted statistical distortion, recovering a "PDOS" that resembles our expectation for the ground state PDOS. 
References:

[1] O. L. Krivanek, T. C. Lovejoy, N. Dellby, T. Aoki, R. W. Carpenter, P. Rez, E. Soignard, J. Zhu, P. E. Batson, M. J. Lagos, R. F. Egerton, and P. A. Crozier, Nature, 514 (2014) 209-212.

[2] P. Rez, T. Aoki, K. March, D. Gur, O. L. Krivanek, N. Dellby, T. C. Lovejoy, S. G. Wolf, and H. Cohen, Nature Communications, 7 (2016) 10945.

[3] M. J. Lagos, A. Trügler, U. Hohenester, and P. E. Batson, Nature, 543 (2017) 529 - 532.

[4] B. D. Forbes and L. J. Allen, Phys. Rev. B, 94 (2016) 014110.

[5] D. Pines and P. Nozieres, The theory of quantum liquids (W. A. Benjamin, New York, 1966).

[6] J. A. Bradley, G. T. Seidler, G. Cooper, M. Vos, A. P. Hitchcock, A. P. Sorini, C. Schlimmer, and K. P. Nagle, Phys. Rev. Lett., 105 (2010) 053202.

This work is supported in part by the Basic Energy Sciences Division of the Department of Energy, Award \#DE-SC0005132.

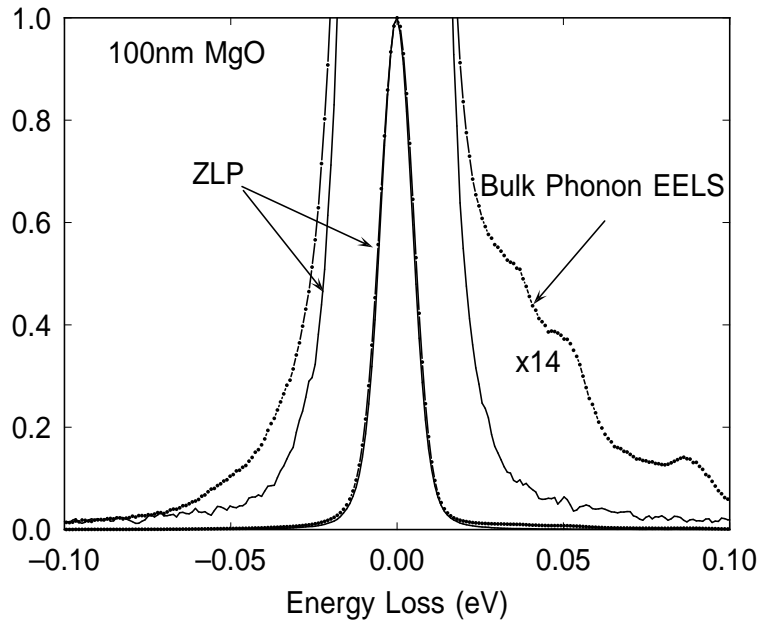

Fig. 1. Vacuum ZLP and bulk data from a $100 \mathrm{~nm} \mathrm{MgO}$ cube normalized at zero energy. Phonon intensities are about $7 \%$ of the total peak intensities. Phonon structure to the right is mainly acoustic modes that are normally not visible to photon absorption experiments.

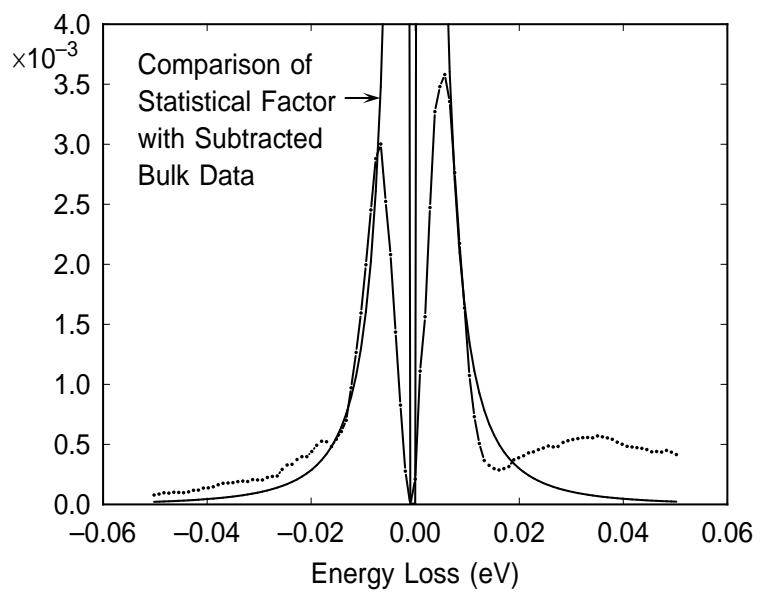

Fig. 3. Subtracted phonon signal is compared with the expected statistical enhancement of PDOS, including a 1 /energy weighting included in $S(\mathbf{q}, \omega)$. [6]

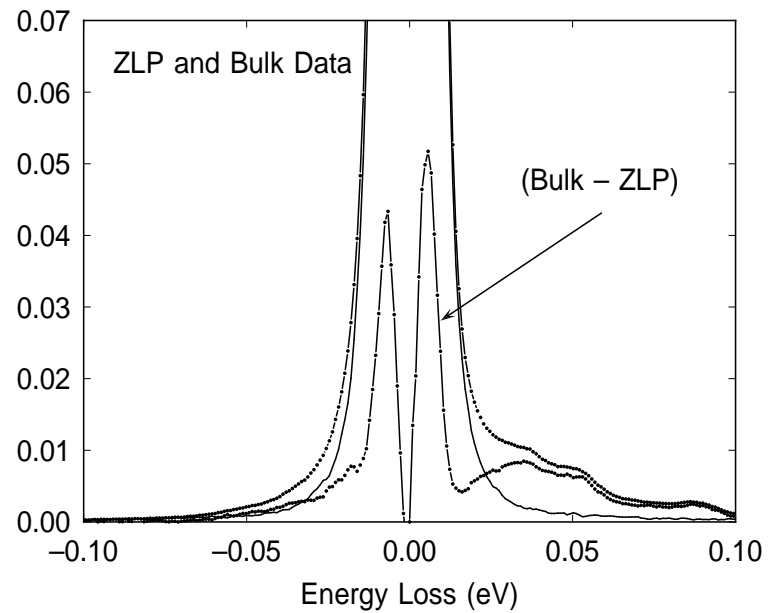

FIG. 2. Phonon signal after subtraction of the measured ZLP. Strong peaks in the $15-25 \mathrm{meV}$ range appear to be phonon scattering. Prima facia evidence for this is the relative intensity of the energy loss and energy gain sides of the ZLP.

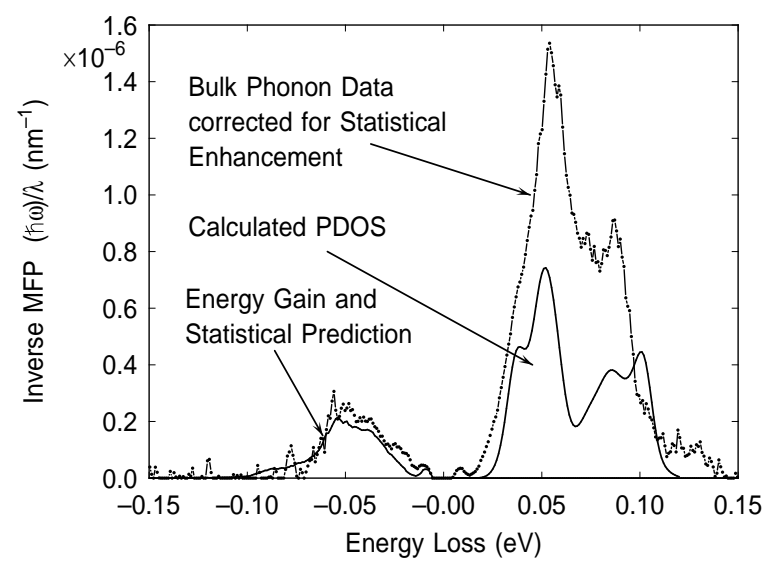

FIG. 4. Final "ground state" estimate after division correction of intensities using the Corrected for Boson Occupation Statistics and numbered in units of Phonon Density of States. 